$\xi=-1$

\title{
Utilizing image processing techniques for detecting breast abnormalities in thermography images
}

\author{
Rabab Saadoon Abdoon* \\ Department of Physics, College of Science, University of Babylon, Hilla, Iraq \\ *Corresponding author E-mail: sr614@ymail.com
}

\begin{abstract}
Thermal Infrared (TIR) imaging of breasts involves a non-invasive, non-ionized, passive, safe and painless scan of the breasts. It is a graphing of the changes in breasts skin temperature using thermography. Thermograms are temperature distribution patterns with different colors to indicate temperature of the different regions within the tested breast, each color refers to a certain temperature range. In this work, three breast thermography images: one for normal case and two for cancerous cases, were employed to test the performance of the proposed segmentation methods: Region growing; clustering (K-means and FCM) algorithms and Histogram based enhancement technique to segment, detect and isolate the suspicious abnormal regions. These techniques were performed with the aid of suitable morphological operations to get the refined regions of interest. The results proved the efficiency of the proposed techniques to extract the abnormal (of high temperature) regions.
\end{abstract}

Keywords: Breast Abnormalities; Histogram Equalization; Segmentation; Temperature Distribution; Thermography.

\section{Introduction}

Many types of examining techniques for breast diseases diagnostic purposes are available including: Mammography, MRI, CT scan, Ultrasound, Thermal IR imaging (Thermography) and PET imaging. Mammography screening is the most popular testing technique, but this technique is not suitable for women aged younger than 40 years and women with dense beasts. As well as, it is invasive examining method utilizes an ionizing radiation (X-rays) and causes discomfort feeling due to the high compression of the patient breasts [1].

Thermography imaging technique can detect exceeding temperature regions (hot spots) that belong to pre-cancerous and cancerous tissues of breast, where these spots associated with high blood vessels and chemical changes [1], [2]. The principle behind this is that, cancerous cells need a large amount of nutrient for their exceeding growth and as a result, the metabolic activity becomes higher in their surrounding area. So, the temperature will be higher in this region compared with other regions [3] According to this, tumors can be considered as a heat source that cause temperature increasing in the surrounding area of the cancerous region and this can be seen as a hot spot in IR Thermograms [4]. Consequently, IR Thermograms could be a first indicator for detecting any cells that may form a cancer or the existed cancerous cells, in many cases from (4-10) years before they be detected by any other imaging techniques including mammography [1]. IR Thermography imaging is safe, passive, non- invasive, utilizing non-ionizing radiation sources, causes no pain and comfort to the patient [4]. In IR Thermograms, the thermal information are demonstrated in a pseudo colored image, acts as a heat distribution pattern of the patient skin, in which each color refers to a certain temperature range $[1,2]$.

There are many researchers work on the breast thermograms utilizing different methods of image processing to investigate the existence of abnormal regions within the samples of Thermal IR images of breasts. The methods that employed by them ranged from edge detection, clustering and neural networks, as well as other methods. For more details of these studies see for instance [5-9].

Segmentation techniques. These image were submitted to the proposed segmentation techniques, which are: Region growing, Kmeans and Fuzzy C-mean (FCM) clustering algorithms and histogram based enhancement technique that employed here for segmentation purpose.

\section{Methods}

Three methods were implemented in this work, which are:

\subsection{Region growing method}

Region growing method usually starts by locating some centroids or seeds each of them represents a distinct region in the image. The seeds began to grow until they eventually cover the entire image. The region growing process is controlled by the rules that describe the mechanism of growth and the one that checks the regions' homogeneity at each growth's step [10]. For more details about the theory of Region growing algorithm see [11]. Many studies were employed this algorithm to segment medical images, such as: [10], [12-15]

\subsection{Clustering (K-means and FCM) algorithms}

Clustering represents a process of segmenting and classifying objects in digital images in such a way that, pixels of the same group are more similar (have the same gray levels, intensity or colors within some criteria) one to another than samples in different groups [10]. 
$\mathrm{K}$-means clustering algorithm is a hard scheme of clustering, it attempts to find groups of pixels (called clusters) within the dataset having similar feature vectors such as intensity, gray levels or colors. The number of clusters has to be fixed in prior. The algorithm involves gathering points of the dataset that have similar feature vectors into a single cluster (segment) and gathering the other data points of non-similar feature vectors into different clusters [16].

Fuzzy c-means (FCM) algorithm is a soft scheme of clustering, in which the dataset point (pixel) is allowed to belong to more than one cluster with different probability or membership grade [16]. For more details see [10]. Many researchers had successfully employed these algorithms to segment medical images, see for instance: [2], [17-21].

\subsection{Histogram equalization technique}

Histogram equalization Technique is utilized to enhances the contrast of the digital images by transforming the values of the image's intensity, so that the histogram of the output enhanced image approximately agrees or matches a pre-specified histogram [22]. In this work, histogram based enhancement technique is employed to achieve segmentation process to extract the abnormal suspicious regions that own high temperature in the temperature distribution images (IR thermograms). For more details about the theory of Histogram equalization technique see [11].

\section{Experimental dataset}

In this study, three breast IR thermograms were adopted. One image is of a normal case, while the other two images were for abnormal cases. The normal breast thermogram was acquired in 3 July, 2018 from American College of Clinical Thermology [23]. This image shows thermal distribution symmetry without any suspicious vascular patterns or significant thermal findings (abnormality) [23], this normal image is presented here for comparison with abnormal images.

The two abnormal images were acquired in 3 July, 2018 too from the internet website [24]. The first abnormal IR thermography image shows a significant amount of thermo vascular activity in the left breast. The patient was referred back to her doctor for further testing. The biopsy result was positive for inflammatory breast cancer. The second abnormal thermography image for another patient shows a large hot region and engorged blood vessels throughout most of the left breast. The patient was advised for an immediate mammography test and follow-up ultrasound test. The mammogram and ultrasound test detected a suspicious area in the left breast, and the biopsy confirmed that it was a cancer [24].

The two abnormal breast thermograms were employed to test the performance of the proposed techniques for segmenting these images and extracting the suspicious regions. Fig. (1) presents the adopted experimental breast thermograms: first image is for the normal case, second image for the first abnormal case and the third image for the second abnormal case.
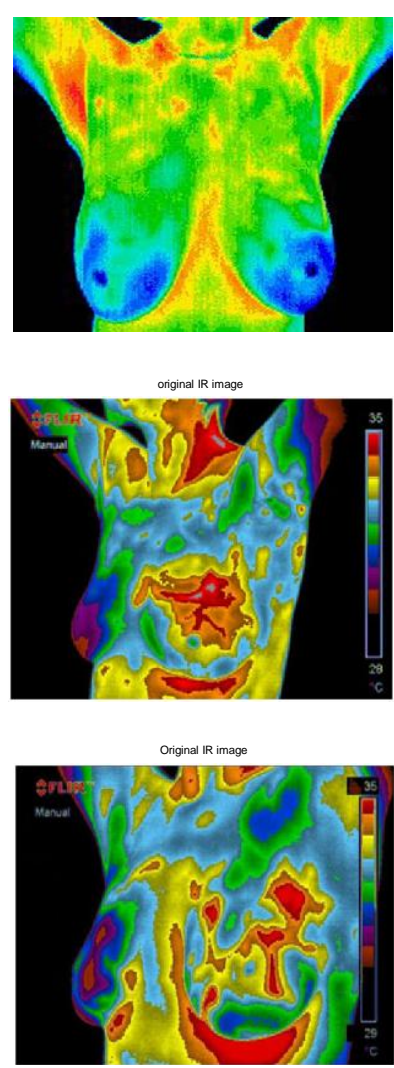

Fig. 1: Shows the Input Breast IR Thermograms.

\section{Experiments and methodologies}

The procedure of this work involves implementing the proposed segmentation techniques, the block diagram of Fig. 2 summarizes this procedure.

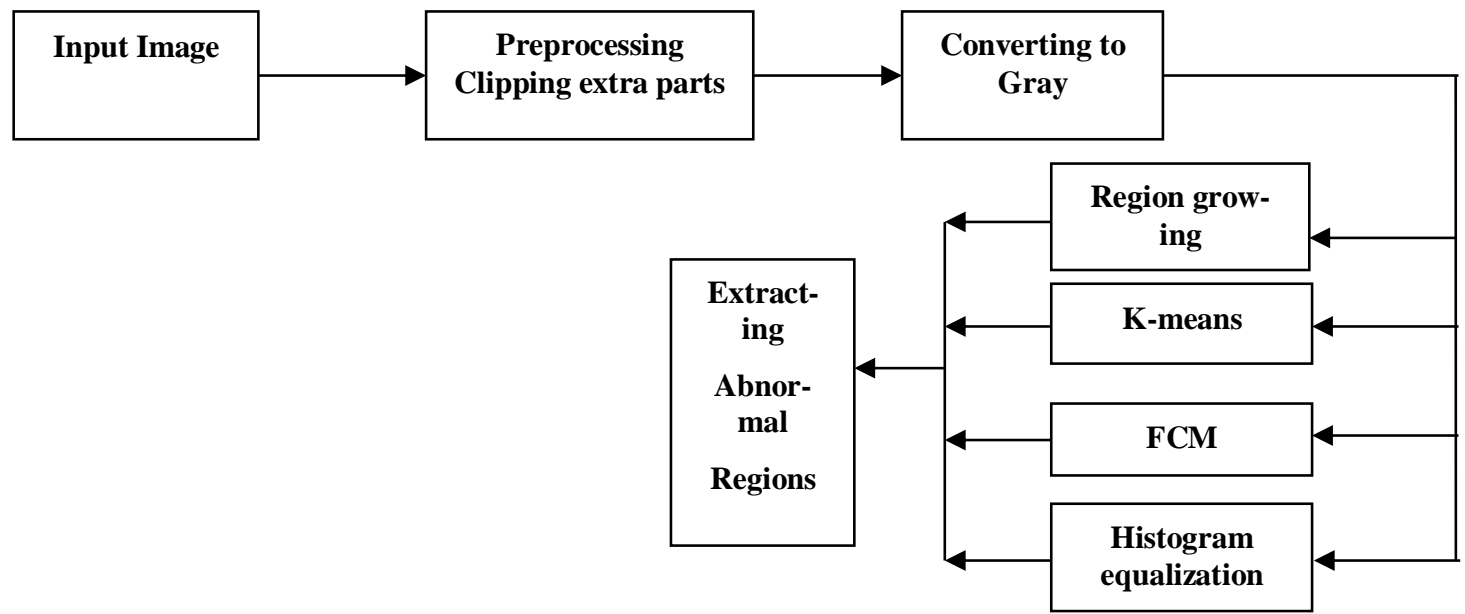

Fig. 2: Block Diagram Illustrates the Proposed Experimental Procedure. 


\section{Results and discussion}

The results of this study, that presented in the procedure of Fig. 2, are demonstrated in the following steps:

\subsection{Preprocessing step: clipping the extra parts}

In this step of the work, the extra parts which are shoulders, neck and the upper part of the abdomen were clipped to process the breasts region only in order to minimize the calculations and to eliminate the processing time as well as to avoid the similarity among the intensity distribution of the breasts and other extra parts. The second operation in this step is to convert the input colored images into gray form to facilitate the segmentation process. The results of this step are presented in Figs. 3 to 5 for the three images respectively.
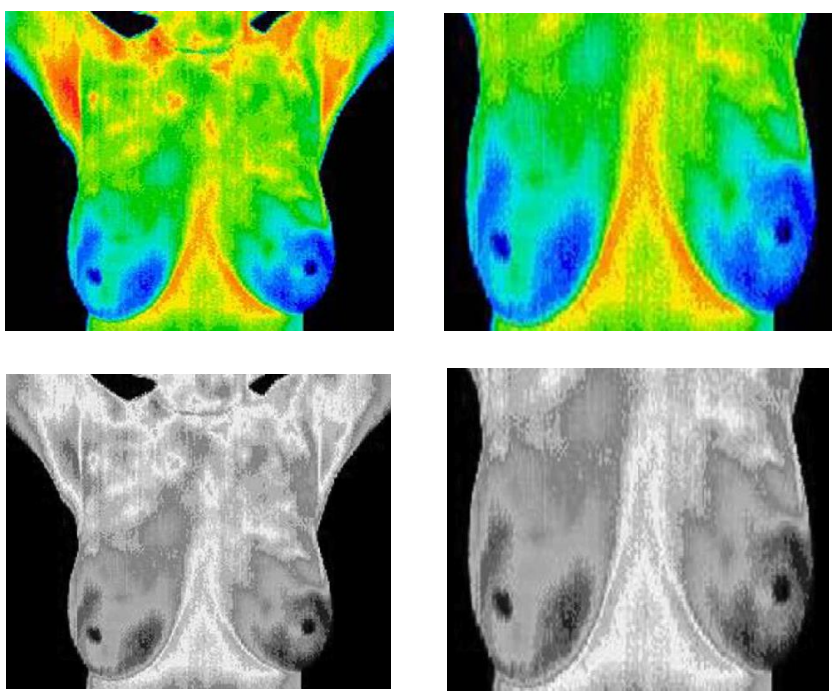

Fig. 3: Thermal IR Input Normal Image: First Line Presents Colored Images Before and After Cutting Extra Body's Parts. Second Line Shows Gray Level Converted Images.
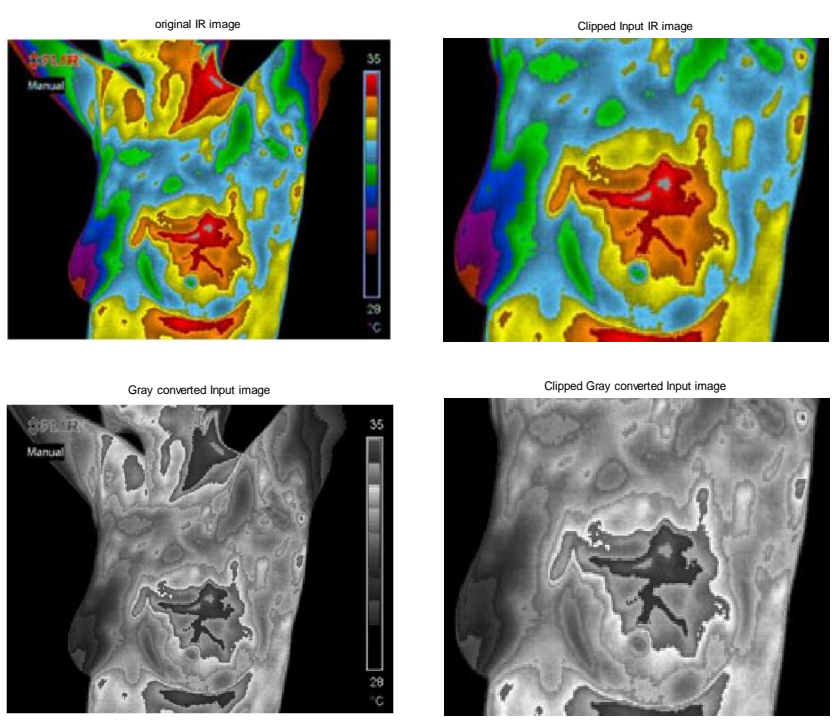

Fig. 4: Thermal IR Input First Abnormal Image: First Line Presents Colored Images Before and After Cutting Extra Body's Parts. Second Line Shows Gray Level Converted Images.
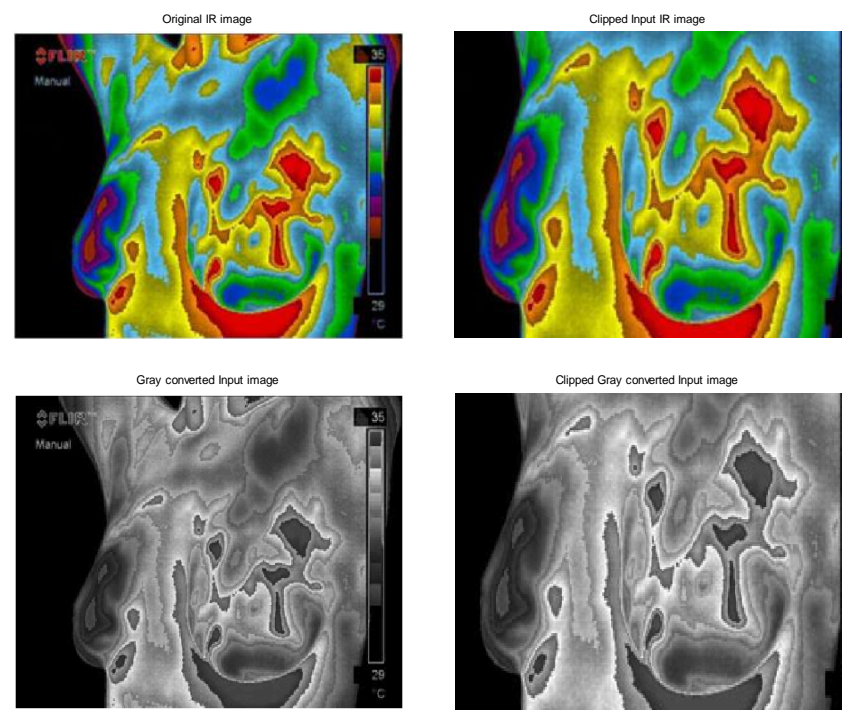

Fig. 5: Thermal IR Input Second Abnormal Image: First Line Presents Colored Images Before and After Cutting Extra Body's Parts. Second Line Shows Gray Level Converted Images.

The original input images were of sizes $600 \times 475 ; 304 \times 231$ and $304 \times 228$ pixels for normal, first abnormal and second abnormal images respectively. After clipping undesired parts, the images sizes became: $457 \times 266 ; 201 \times 141$ and $245 \times 153$ pixels for the three images respectively.

\subsection{Segmentation step}

In this step of the work, four techniques were implemented to segment the adopted abnormal breast IR thermograms.

\subsubsection{Region growing method}

Region growing, which is region based segmentation method, was implemented on the two abnormal thermograms with different differentiation threshold values ranged from 10 to 60 with steps of 5. The results for the first abnormal image are presented in Fig. 6. From the results, it was found that the value 60 is a suitable differentiation threshold value to help extracting abnormality region after applying contouring process to the resultant region growing segmented images. 

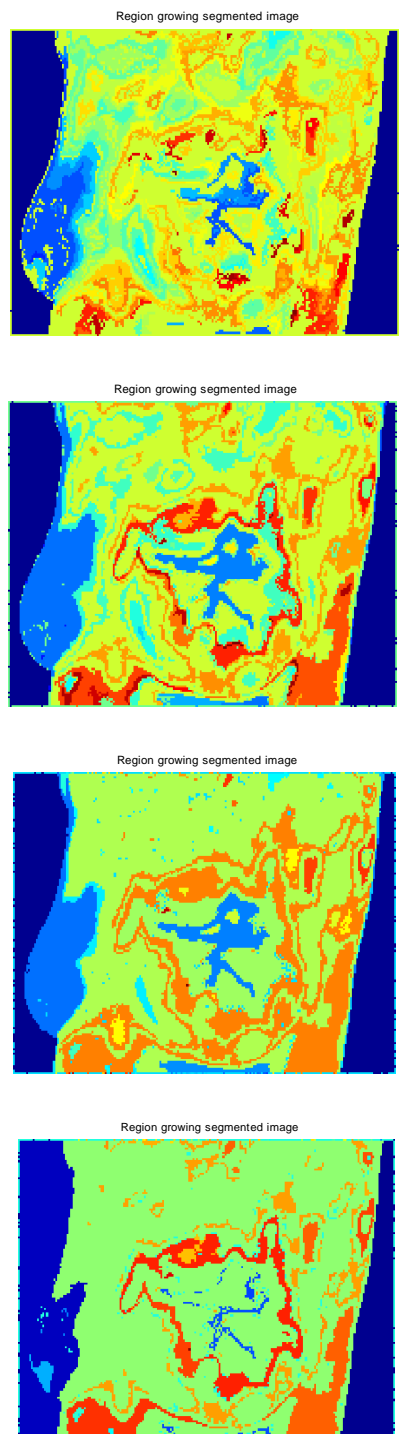
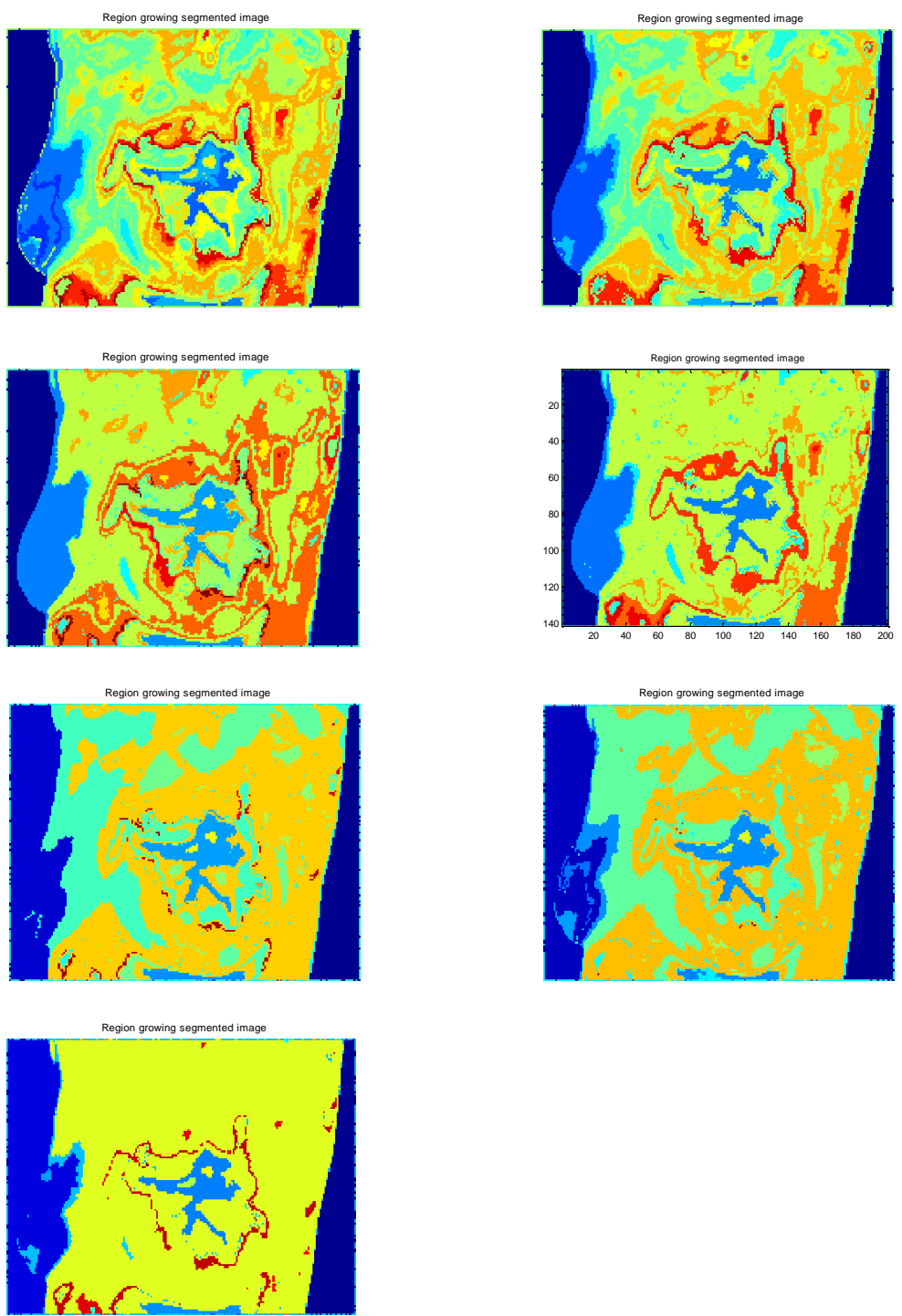

Fig. 6: Region Growing Segmented First Abnormal Image with Differentiation Threshold Values: 10 to 60 With Steps of 5 from First Image in First Row to the Last One Respectively.

Table 1: Elapsed Time of Implementing Region Growing Method with Different Values of Differentiation Threshold

\begin{tabular}{|c|c|c|c|c|c|c|c|c|c|c|c|}
\hline Threshold value & 10 & 15 & 20 & 25 & 30 & 35 & 40 & 45 & 50 & 55 & 60 \\
\hline Elapsed time (sec) & 255.97 & 139.84 & 110.74 & 90.61 & 63.96 & 62.17 & 49.04 & 49.09 & 51.70 & 47.16 & 38.10 \\
\hline
\end{tabular}

The elapsed time of implementing Region growing method with different values of differentiation threshold (10-60) with steps of 5 was calculated. These times are presented in Table 1, it is cleared that, elapsed time decreases as the differentiation threshold value increases.

The steps of extracting abnormality (high temperature) region of the two abnormal input image by implementing Region growing method with differentiation threshold equals 60 followed by contouring process are illustrated in Figs. 7 and 8 for first and second abnormal images respectively.

\section{(A)}

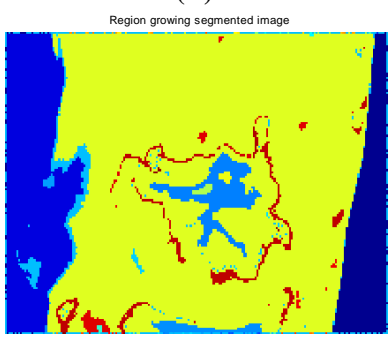

(B)

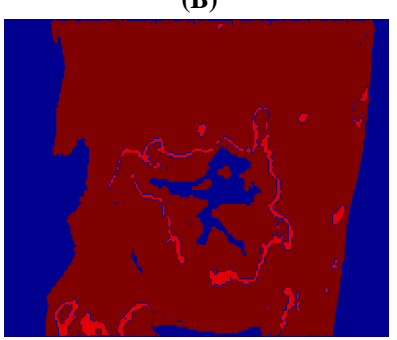

(C)

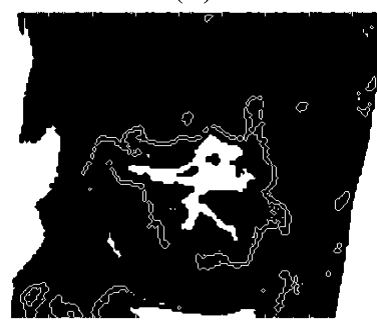

(E)

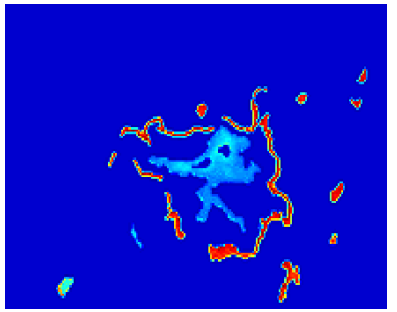

Fig. 7: Steps of Extracting Abnormality (High Temperature) Region of the First Abnormal Input Image by Implementing Region Growing Method with Differentiation Threshold Equals 60. (A) Segmented Image; (B) 
Contoured Image of Image A; (C) B/W Image; (D) Extracted Abnormal Region and (E) Colored Image of Image D.

(A)

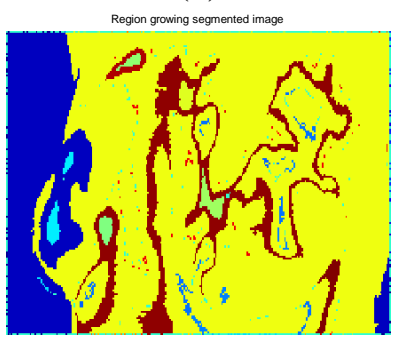

(C)

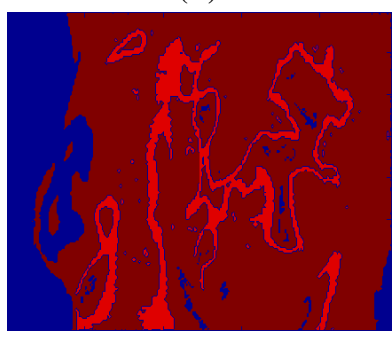

(E)

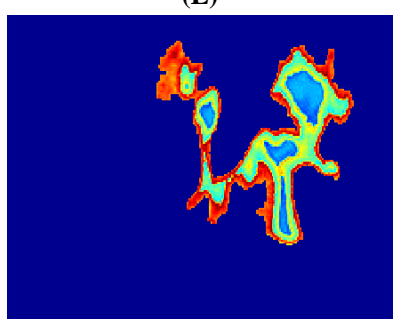

(B)

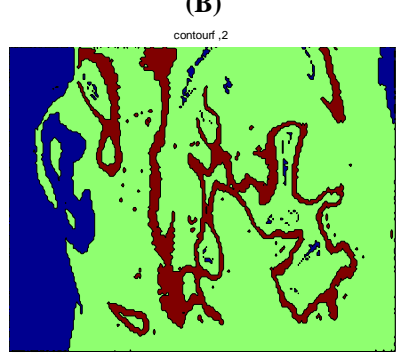

(D)
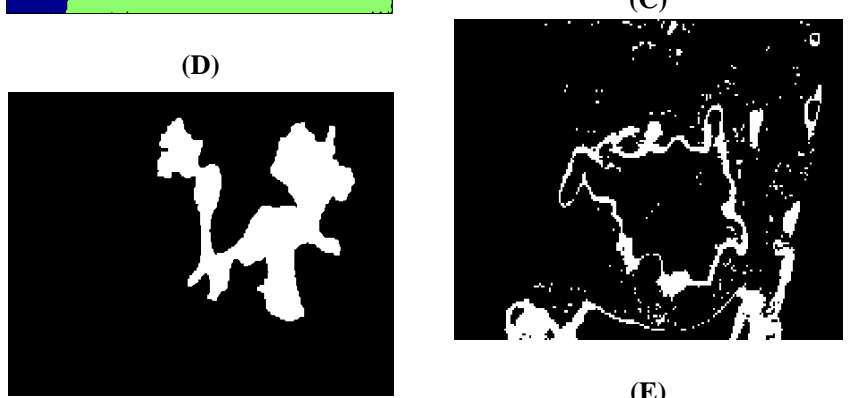

(E)

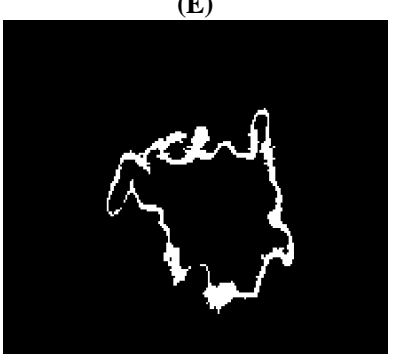

(G)

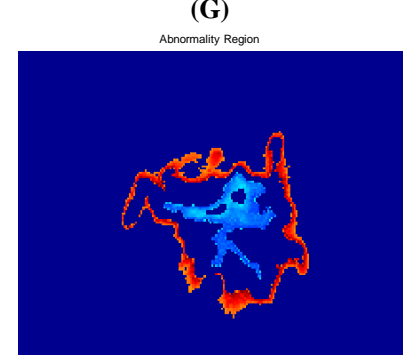

Fig. 9: Steps of Extracting Abnormality (High Temperature) Region of the First Input Image by Implementing K-Means Algorithm with Five Clusters. (A) Segmented Image; (B) Cluster of Inner Abnormal Region; (C) Cluster of Outer (Surrounding) Abnormal Region; (D) Extracted Inner Abnormal Region; (E) Extracted Outer (Surrounding) Abnormal Region; (F) Whole Extracted Abnormal Region and (H) Colored Image. converted image of B image; (D) shows the extracted abnormal region and (E) presents the colored image. Region growing method followed by contouring process proved its efficiency to segment breast thermography images.

\subsubsection{K-means algorithm}

K-means algorithm with different number of clusters is employed to cluster the adopted two breast thermograms. The results showed that, the number five is the proper number of clusters to segment these two abnormal breast IR image. The results of implementing K-means on the first and second experimental abnormal images with 5 clusters followed by morphological suitable processes are presented in Figs 9 and 10 respectively. 
(A)

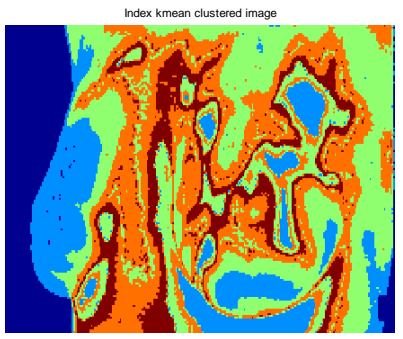

(C)

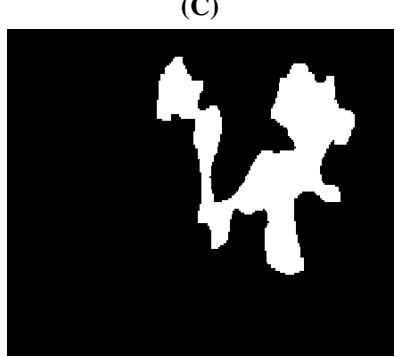

(B)

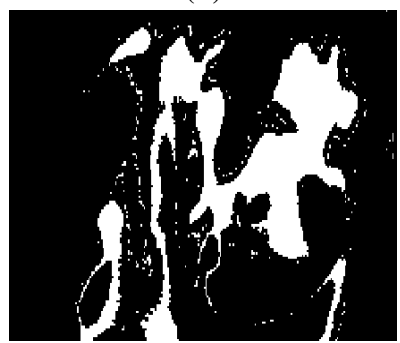

(D)

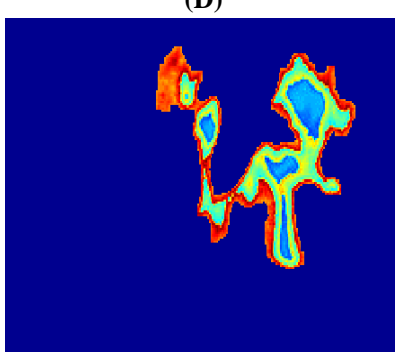

Fig. 10: Steps of Extracting Abnormality (High Temperature) Region of the Second Abnormal Input Image by Implementing K-Means Algorithm with Five Clusters. (A) Segmented Image; (B) Cluster of Abnormal Region; (C) Extracted Abnormal Region and (D) Colored Extracted Abnormal Region.

Fig. 9: shows the steps of extracting abnormality (high temperature) region of the first abnormal input image by implementing $\mathrm{K}$ means algorithm with five clusters. (A) shows the segmented image; (B) represents the cluster of inner abnormal region; (C) presents the cluster of outer(surrounding) abnormal region;(D) shows the extracted inner abnormal region; (E) shows the extracted outer(surrounding) abnormal region; (F) represents the whole extracted abnormal region and $(\mathrm{H})$ represents the colored image.

Fig. 10: presents the results of the steps of extracting abnormality (high temperature) region of the second abnormal input image utilizing K-means algorithm with five clusters. (A) shows the segmented image; (B) represents the cluster of abnormal region; (C) shows the extracted abnormal region while (D) represents the colored extracted abnormal region. It is clear that, K-means algorithm is an efficient method to segment and extract suspicious abnormal regions in breast IR thermograms.

\subsubsection{FCM algorithm}

After trying many number of clusters, it is found that, the number five clusters is proper to segment the two abnormal breast thermal IR images by utilizing FCM algorithm. The results of implementing FCM on the first and second abnormal thermography images with 5 clusters are presented in Figs 11 and 12 respectively.

(A)

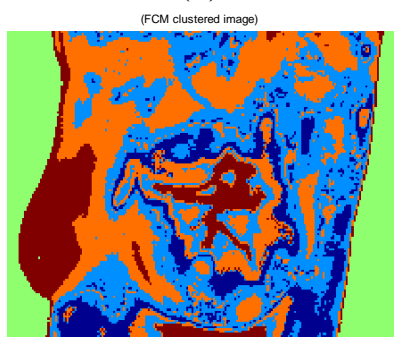

(B)

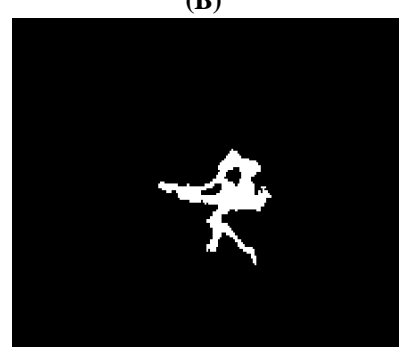

(C)

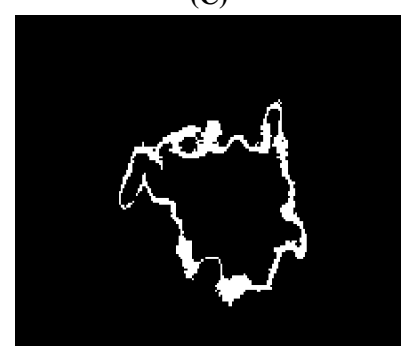

(E)

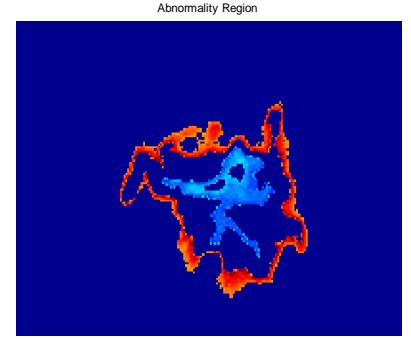

Fig. 11: Steps of Extracting Abnormality (High Temperature) Region of the First Abnormal Input Image by Implementing FCM Algorithm with Five Clusters. (A) Segmented Image; (B) Extracted Inner Abnormal Region Image; (C) Extracted Outer (Surrounding) Abnormal Region; (D) Whole Extracted Abnormal Region and (E) Colored Whole Extracted Abnormal Region.
(A)

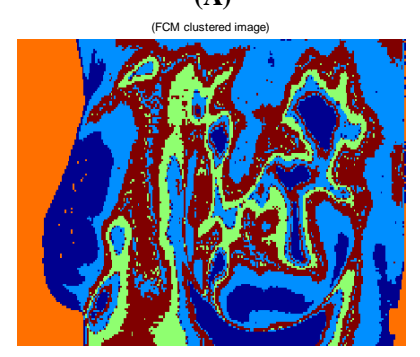

(C)

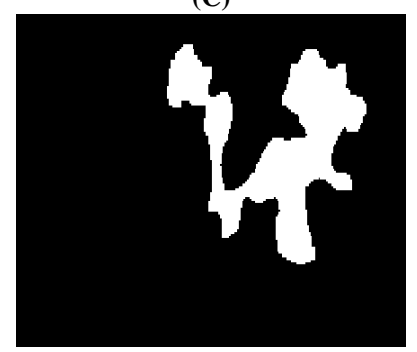

(B)

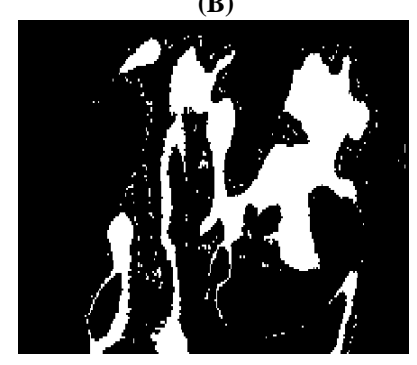

(D)

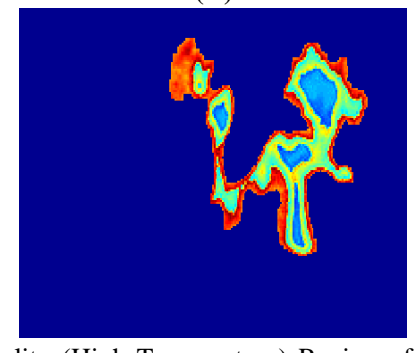

Fig. 12: Steps of Extracting Abnormality (High Temperature) Region of the Second Abnormal Input Image by Implementing FCM Algorithm with Five Clusters. (A) Segmented Image; (B) Cluster of Abnormal Region; (C) Extracted Abnormal Region and (D) Colored Extracted Abnormal Region.

In Fig. 11, the steps of extracting abnormality (high temperature) region of the first abnormal input image by implementing FCM algorithm with five clusters are illustrated. (A) represents the segmented image; (B) shows the extracted inner abnormal region image; (C) presents the extracted outer(surrounding) abnormal region; (D) shows the whole extracted abnormal region and (E) represents the colored whole extracted abnormal region.

Fig. 12 illustrates the steps of extracting abnormality (high temperature) region of the second abnormal input image by implementing FCM algorithm with five clusters. (A) shows the segmented image; $(\mathrm{B})$ represents the cluster that the abnormal region belong to; (C) presents the extracted abnormal region while (D) presents the colored extracted abnormal region .

From Figs. 11 and 12, it is clear that, FCM algorithm succeeded to detect and extract the suspicious abnormal regions. 


\subsubsection{Histogram equalization}

In this step of the work, Histogram equalization enhancement technique utilized here for segmentation purpose. This technique was implemented to extract the abnormal suspicious region in the first abnormal thermogram. The results of applying this technique with the aid of suitable morphological operations to get refine abnormal suspicious region, is demonstrated in Fig. 13.
(A)

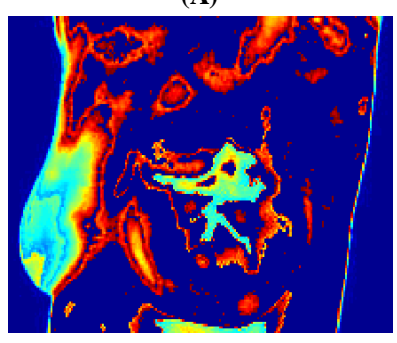

(C)

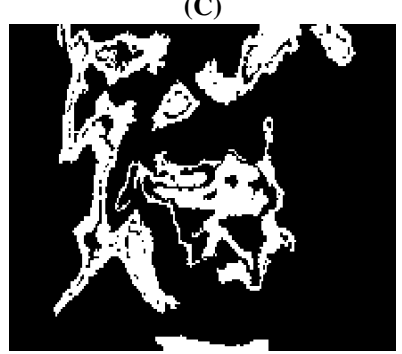

(E)

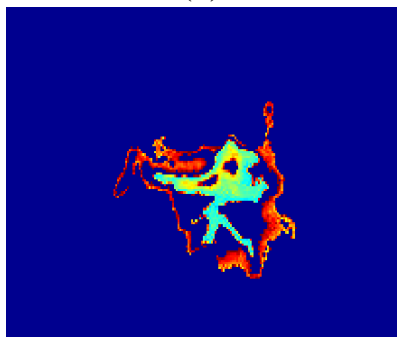

(B)

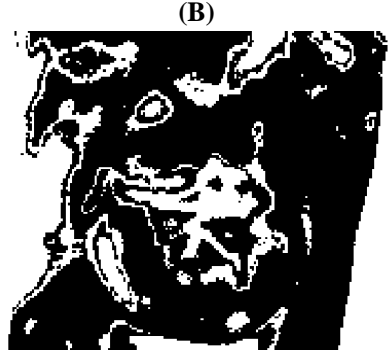

(D)

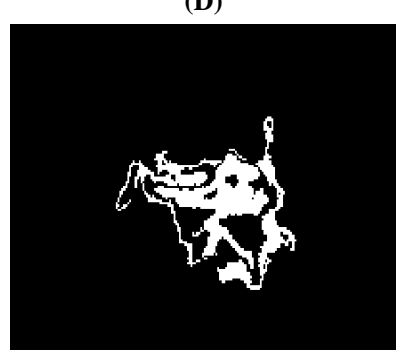

Fig. 13: Steps of Extracting (High Temperature) Region of the First $\mathrm{Ab}$ normal Input Image by Implementing Histogram Equalization Technique. (A) Histogram Equalized Image; (B) B/W Image of the Previous Image A; (C) Background Disregarded Image; (D) Extracted Abnormal Region and (E) Colored Extracted Abnormal Region .

Fig. 13 shows the steps of extracting abnormal region in first abnormal thermogram, it is cleared that, this technique succeeded robustly to isolate and extract the suspicious abnormal region.

\section{Conclusion}

In this work, three breast IR thermograms, one for normal case and two for cancerous cases, were employed to test the performance of the proposed segmentation methods: Region growing; clustering (K-means and FCM) algorithms and Histogram equalization, which is histogram based enhancement techniques to segment, detect and isolate the suspicious abnormal regions. These techniques were performed with the aid of suitable morphological operations to get the refined regions of interest. From the results one can deduced that, Region growing method followed by contouring process proved its efficiency to segment breast thermography images after finding out the proper differentiation threshold value which equals 60; clustering K-means and FCM algorithms succeeded to perform good quality segmenting process after finding that 5 clusters is the suitable number of clusters to segment breast thermograms; as well as, Histogram equalization enhancement technique was a robust method to extract the abnormal suspicious region.
The results proved the efficiency of the four proposed techniques to extract the abnormal regions that own high temperature in the temperature distribution graphs (thermograms) of breasts.

\section{References}

[1] P. Kapoor, E. Bhayana and S.V.A.V Prasad. Real Time Intelligent Thermal Analysis Approach for Early Diagnosis of Breast Cancer. Vol.1, No. 5(2011)22-24

[2] N. Golestani, M. Etehad Tavakol, and E. Y. K. Ng, Level Set Method for Segmentation of Infrared Breast Thermograms, EXCLI Journal, Vol.13, (2014).241-251.

[3] N. Selvarasu, A. Nachaippan and N.M. Nandhitha. Image Processing Techniques and Neural Networks for Automated Cancer Analysis from Breast Thermographs-A Review. Indian Journal of Computer Science and Engineering, Vol. 3, No. 1 (2012) 133-137.

[4] V. Umadevi, S.V. Raghavan and S. Jaipurkar. Framework for estimating tumour parameters using thermal imaging. Indian $\mathrm{J}$ Med Res 134, (2011)725-731. https://doi.org/10.4103/0971-5916.91012.

[5] H. Q. Yang, S.-S. Xie, Q.-Y. Lin, Z. Ye, S.-Q. Chen, H. Li. A new infrared thermal imaging and its preliminary investigation of Breast Disease Assessment, Proc. Of IEEE/ICME, International Conference on Complex Medical Engineering, (2007) 1071-1074.

[6] X. Tang, H. Ding, Y. Yuan, Q. Wang. Morphological Measurement of Localised temperature Increase Amplitudes in breast Thermograms and its clinical application. Biomedical Signal processing

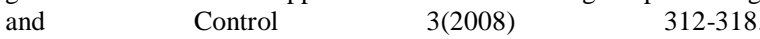
https://doi.org/10.1016/j.bspc.2008.04.001.

[7] H. G. Zadeh, I. A. Kazerouni and J. Haddadin. Distinguish breast cancer based on thermal Features in Infrared Images, Canadian Journal on Image processing and computer vision, Vol.2, No.6 (2011) 54-58.

[8] N. A. Mohamed. Breast cancer risk detection using digital infrared thermal images. International Journal of Bioinformatics and Biomedical Engineering, Vol. 1, No. 2 (2015) 185-194.

[9] A. A. Al Dergazly. Thermal images segmentation for evaluation of breast cancer. International Journal of Science and Research, Vol. 6 Issue 2 (2017) 230-242.

[10] R. S. Abdoon, L. K. Abood and S. M Ali. Adaptive techniques for brain tumor detection in MRI", Lap LAMBERT Academic Publishing, Germany (2015)

[11] R. C. Gonzalez and R. E Woods. Digital image processing. Prentice Hall, New Jersey, (2008).

[12] T. Y. Law and P. A. Heng. Automated extraction of bronchus from 3D CT images of lung based on genetic algorithm and 3D region growing. Medical Imaging Proc. of SPIE, Vol. 3979 (2000)906916.

[13] M. D. Noorul Mubarak, M. M. Sathik, Z. S. Beevi and K. Revathy. A hybrid region growing algorithm for medical image segmentation. International Journal of Computer Science \& Information Technology (IJCSIT), Vol. 4, No. 3(2012) 61-70. https://doi.org/10.5121/ijcsit.2012.4306.

[14] W. A. Abbas. CT-scan lung cancer detection using image processing techniques. Master Thesis in image processing and remote sensing, University of Baghdad, Iraq (2014).

[15] R. S. Abdoon. Region growing and k-means hard clustering technique to extract brain abnormalities in MRI images. Journal of Babylon University/Pure and Applied Sciences, Vol. 25, No. 3, (2017)1077- 1087

[16] R. S. Abdoon and O. S. Khudair. Implementing segmentation techniques to isolate brain tumors in CT scan and MRI images for different orientations and modalities. Journal of Advanced Microscopy Research, Vol. 12, Issue 2(2017)116-129. https://doi.org/10.1166/jamr.2017.1329.

[17] D. Q. Zhang, S. C. Chen, Z. S. Pan and K. R. Tan. Kernel- based fuzzy clustering incorporating spatial constraints for image segmentation. Proc. International Conference on Machine Learning and Cybernetics, Vol. 4(2003) 2189-2192

[18] H. P. Ng, H. H. Ong, K. W. C. Foong, P. S. Goh and W. L. Nowinski. Medical image segmentation using k-means clustering and improved watershed algorithm. IEEE Southwest Symposium on Image Analysis and Interpretation (2006) 61-65 https://doi.org/10.1109/SSIAI.2006.1633722.

[19] Y. Yang and S. Huang. Image segmentation by fuzzy c-means clustering algorithm with a novel penalty term. Computing and Informatics, Vol. 26 (2007) 17-31.

[20] K. S. Angel Viji and J. Jayakumari. Performance evaluation of standard image segmentation methods and clustering algorithms for 
segmentation of MRI brain tumor images. European Journal of Scientific Research, Vol. 79, No. 2 (2012) 166-179.

[21] P. Dhanalakshmi and T. Kanimozhi. Automatic segmentation of brain tumor using $\mathrm{k}$-means clustering and its area calculation. International Journal of Advanced Electrical and Electronics Engineering, Vol. 2, Issue 2 (2013) 130-134.

[22] R. C Gonzalez, R. E. Woods and S. L. Eddins. Digital image processing using MATLAB. Pearson Prentice-Hall (2004).

[23] American College of Clinical Thermology, available online at: http://www.thermologyonline.org/Breast/breast_thermography_wha t.htm, accessed July 3, 2018.

[24] Available online at Website: http://www.breastthermography.com/case_studies.htm, accessed July 3, 2018 